\title{
Heridas múltiples penetrantes intracraneales causadas por pistola de clavos: caso clínico
}

\author{
I. Zazpe; A. Vázquez; C. Beaumont*; A. Bardón*; J. Azcona; A. Gallo-Ruiz y E. Portillo
}

Servicios de Neurocirugía y de Urgencias*. Hospital de Navarra.

\section{Resumen}

Las heridas penetrantes intracraneales son muy infrecuentes y aún resultan más infrecuentes las causadas por múltiples objetos y de forma intencional. Presentamos el caso de un paciente varón de 45 años que es traído a urgencias tras haberse disparado cuatro clavos a nivel del cráneo y uno a nivel del tórax con una pistola de clavos. Revisamos la literatura al respecto y describimos cómo fue manejado el caso en nuestro centro con buen resultado funcional.

PALABRAS CLAVE: Herida penetrante intracraneal. Pistola de clavos. Cuerpo extraño intracraneal.

Multiple penetrating brain injuries caused by a nail gun: a case report

\section{Summary}

Multiple and self-inflicted penetrating brain injuries (PBI) rare. $\mathrm{PBI}$ are rare. We report the case of multiple self-inflicted PBI in a 45 year-old man caused by a nail gun. He was admitted to after shooting four nails into his head and one into his thorax. We review the literature on the topic and describe how this case was successfully managed in our hospital.

KEY WORDS: Penetrating brain injury. Nail gun. Intracraneal foreign body.

\section{Introducción}

Se consideran heridas intracraneales penetrantes ("penetrating brain injury", PBI) a aquéllas que presentan únicamente orificio de entrada, en contraposición a las heridas perforantes (transfixiantes), que son aquéllas con orificio de entrada, y de salida. Las PBI constituyen una pequeña parte de los traumatismos intracraneales. Sin embargo, su

Recibido: 16-06-05. Aceptado: 18-09-05 incidencia está aumentando (especialmente las producidas por arma de fuego) y su morbimortalidad continúa siendo muy elevada. El pronóstico de las PBI depende del tipo de proyectil o instrumento causante, la energía del impacto, la localización y la trayectoria intracraneal. Además de la lesión cerebral primaria secundaria al impacto inicial, pueden desencadenarse lesiones secundarias más tardías.

Las PBI pueden estar causadas por instrumentos introducidos localmente o lanzados a distancia (tipo proyectil). Las heridas por proyectiles pueden estar producidas por proyectiles de baja o de alta velocidad. Entre los de baja velocidad se encuentran los producidos por pistolas de clavos, algunas pistolas utilizadas en el ámbito civil y otras pistolas de uso laboral. Entre los proyectiles de alta velocidad se encuentran las balas de armas de fuego. Éstas pueden dañar el parénquima cerebral por tres mecanismos: laceración, cavitación (que puede alcanzar un tamaño hasta 30 veces mayor que el tamaño del proyectil) y ondas de choque, capaces de lesionar estructuras a distancia de la trayectoria del proyectil.

Entre las causadas por objetos no proyectados destacan las producidas por arma blanca. Se caracterizan por su baja velocidad y pequeña superficie de impacto. El objeto más frecuente es el cuchillo, aunque se han descrito casos producidos por enseres variopintos, como palos de golf, palos de hockey, barras de metal, lápices, tijeras,... En este tipo de PBI no existe cavitación ni lesiones primarias a distancia. La existencia de infarto o hematoma asociados empeora notablemente el pronóstico.

El caso que presentamos está causado por proyectiles de baja velocidad (pistola de clavos), que poseen una energía de impacto intermedia entre las heridas causadas por arma blanca y las producidas por arma de fuego. Las heridas por pistolas de clavos suelen causar menor daño

Abreviaturas. CO: monóxido de carbono. GCS: Glasgow Coma Score. HSA: hemorragia subaracnoidea. LCR: líquido cefalorraquideo. PBI: penetrating brain injury, herida penetrante intracraneal. PIC: presión intracraneal. RMN: resonancia magnética nuclear. TC: tomografía computarizada. TCE: traumatismo cráneo-encefálico 

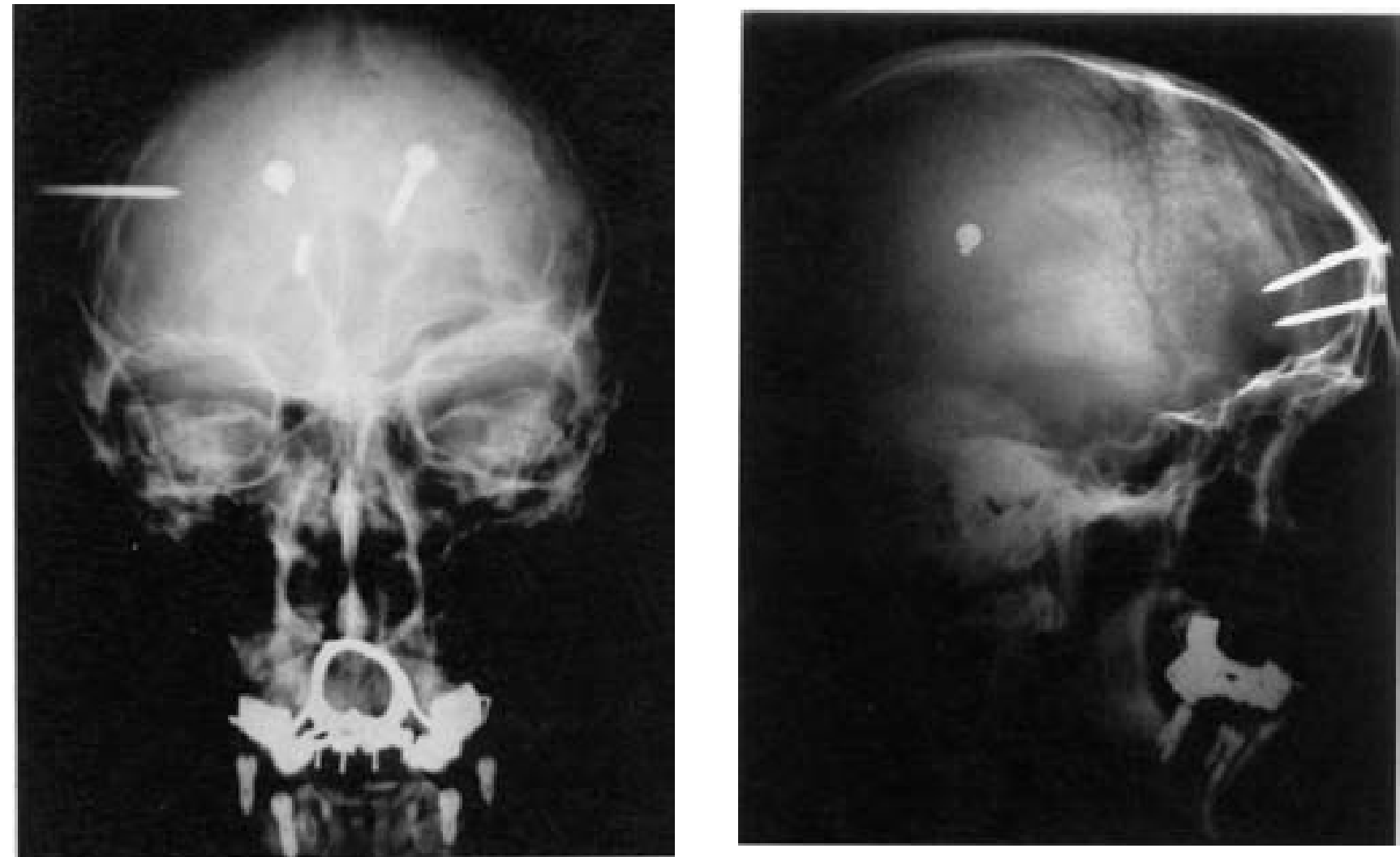

Figuras 1 y 2. Rx AP y lateral de cráneo al ingreso. Se objetivan dos clavos a nivel frontal derecho, uno a nivel temporal derecho y otro a nivel frontal izquierdo.
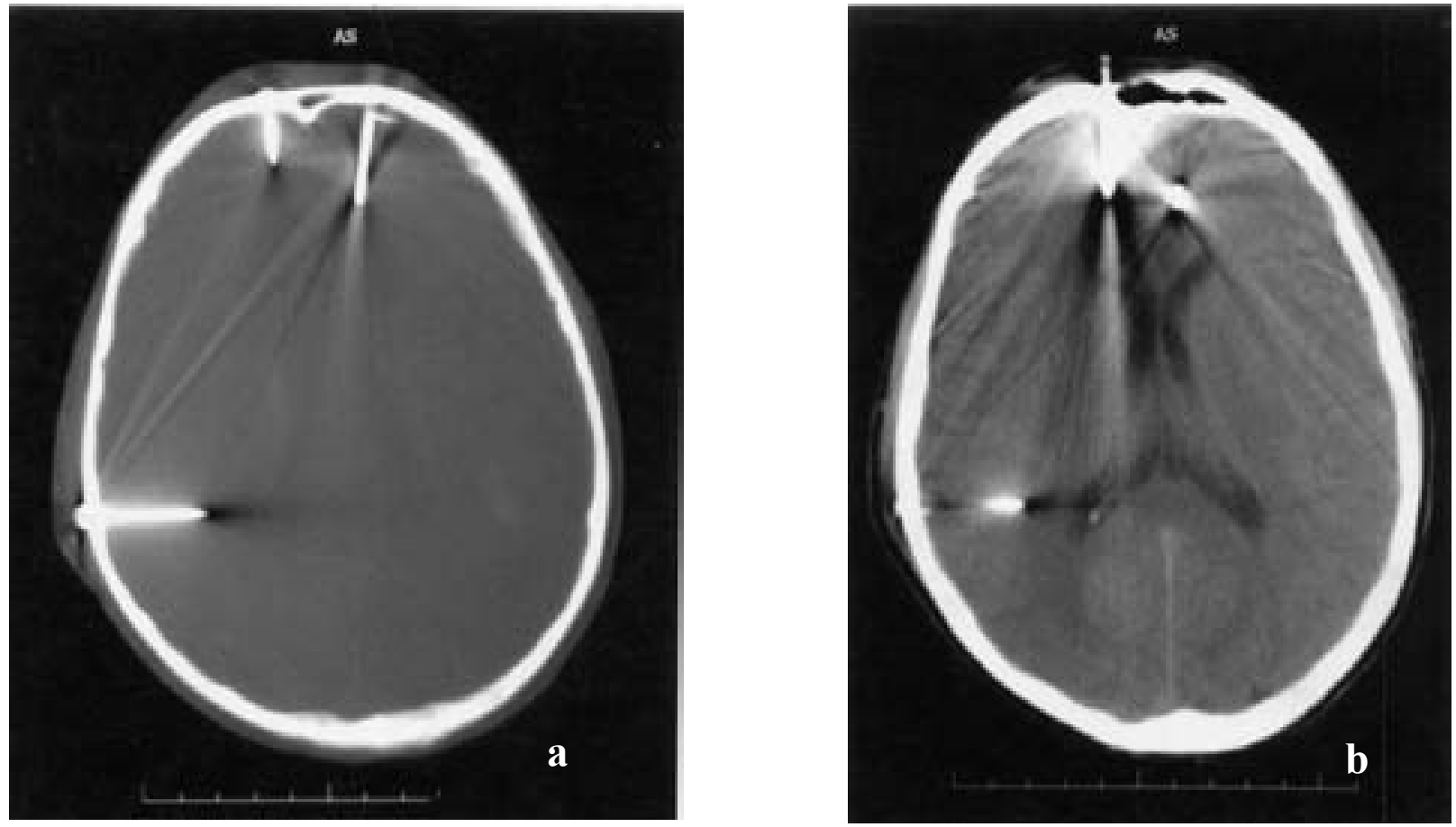

Figura 3. TC craneal al ingreso: $3 a$, ventana ósea y 3b, ventana de parénquima. No se observan hematomas, fracturas ni lesiones intraparenquimatosas. 


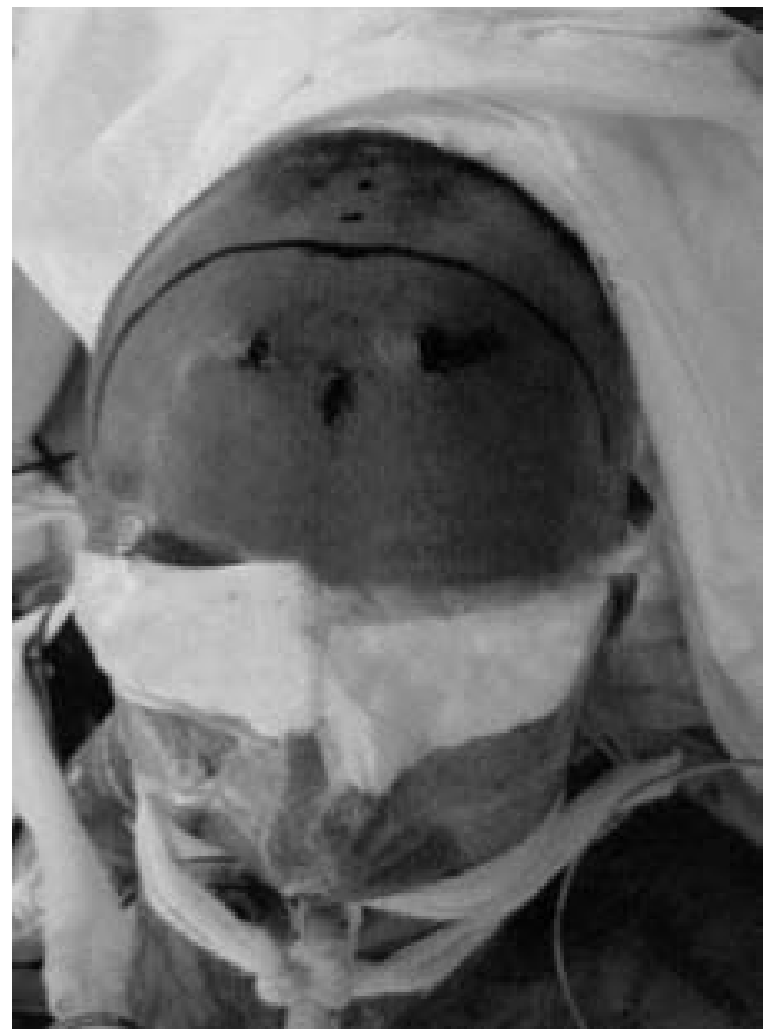

Figura 4. Orificios de entrada de los clavos en región frontal. Planificación de la intervención.

cerebral y tienen mejor pronóstico que las causadas por arma de fuego, sin embargo, la infección intracraneal y la lesión vascular pueden ser complicaciones letales.

\section{Caso clínico}

Paciente varón de 45 años de edad sin antecedentes psiquiátricos ni médicos de interés. Al parecer cuatro días antes llevó a cabo diversos intentos autolíticos que inicialmente no confesó y posteriormente permaneció desaparecido en el monte hasta ser encontrado por un amigo, que lo trajo a urgencias. A su llegada a urgencias el paciente refirió únicamente cuadro de cefalea sin otra explicación. Se encontraba afebril, consciente, orientado, GCS (Glasgow Coma Score) 15 puntos, pupilas isocóricas y normorreactivas y sin déficits neurológicos. Presentaba heridas incisocontusas con costras en varias localizaciones (tres en región frontal, una en región temporal derecha y una en región submamaria izquierda) que no dejaban entrever la presencia de cuerpos extraños en esas localizaciones. En la auscultación se objetivó hipoventilación en base izquierda, siendo el resto de la exploración física normal. Se practicó $\mathrm{Rx}$ tórax que mostró neumotórax izquierdo y cuerpo extraño compatible con clavo a nivel de tercio medio de hemitórax izquierdo. Se entrevistó nuevamente al paciente reconociendo en

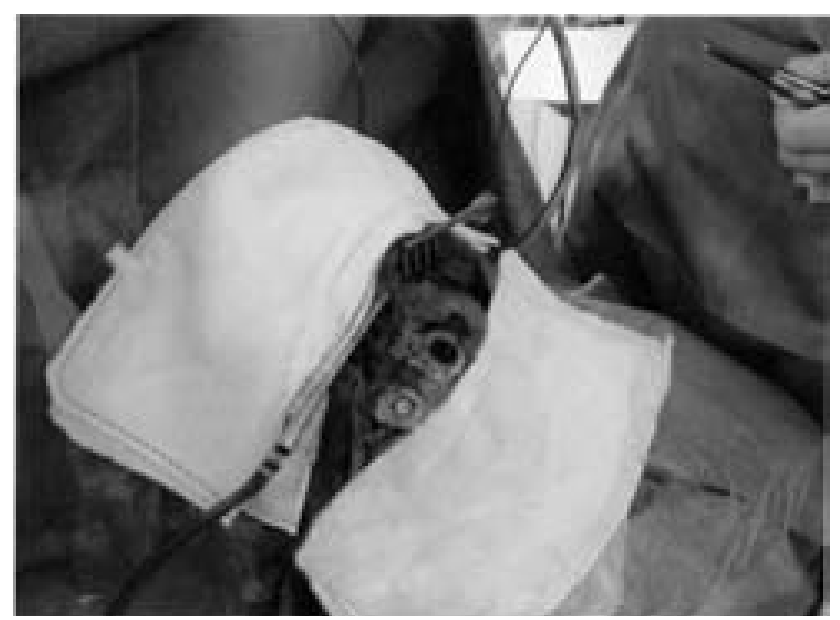

Figura 5. Identificación de los clavos impactados en el diploe frontal. Uno de ellos presenta un recubrimiento de la cabeza con un remache de goma de color negro. Uno de los clavos frontal derecho se encuentra descabezado.

ese momento haberse disparado otros cuatro clavos en la cabeza, por lo que se practicaron $\mathrm{Rx}$ de cráneo y una TC de cráneo. La Rx cráneo (Figs. 1 y 2) objetivó la presencia de cuatro clavos a nivel intracraneal: uno frontal izquierdo de $4 \mathrm{~cm}$, dos frontales derechos de 4 y $2 \mathrm{~cm}$ y uno a nivel temporal derecho de $4 \mathrm{~cm}$. La TC de cráneo (Fig. 3) mostró que ninguno de los clavos había atravesado en su trayectoria estructuras vasculares ni había signos de hemorragia. El paciente fue valorado por el neurocirujano, el cirujano torácico y el psiquiatra de guardia e ingresado en el servicio de observación de urgencias. Se solicitó a la familia una muestra de los clavos utilizados, comprobándose la longitud y estructura de los mismos. Se instauró profilaxis antibiótica con ceftriaxona durante 1 semana. No se instauró profilaxis anticomicial. Tras colocar el drenaje pleural en urgencias no se consiguió expansión pulmonar, por lo que el paciente fue intervenido con carácter urgente por el servicio de cirugía torácica y, seguidamente, por el de neurocirugía en el mismo acto quirúrgico. Se le practicó toracotomía izquierda, extracción de clavo que penetraba en pulmón izquierdo, limpieza y reparación de tejidos. Por parte de neurocirugía se procedió a limpieza de las heridas cutáneas. Tras retirada de capa de costra que las cubría se observaron pequeñas heridas incisocontusas en la piel correspondientes a los orificios de entrada (Fig. 4). Se practicaron dos incisiones: una bicoronal para acceder a los tres clavos de localización frontal (Fig. 5) y otra incisión temporal derecha para acceder al clavo en dicha localización. Una vez se llegó a calota, se localizaron los orificios de entrada de cada uno de los clavos. Se procedió a fresar alrededor de la cabeza impactada en el diploe de cada uno de los clavos hasta visualizar la duramadre y dejar la cabeza móvil. Posteriormente se extrajo el clavo tirando suavemente de él hacia fuera en la misma dirección en la 


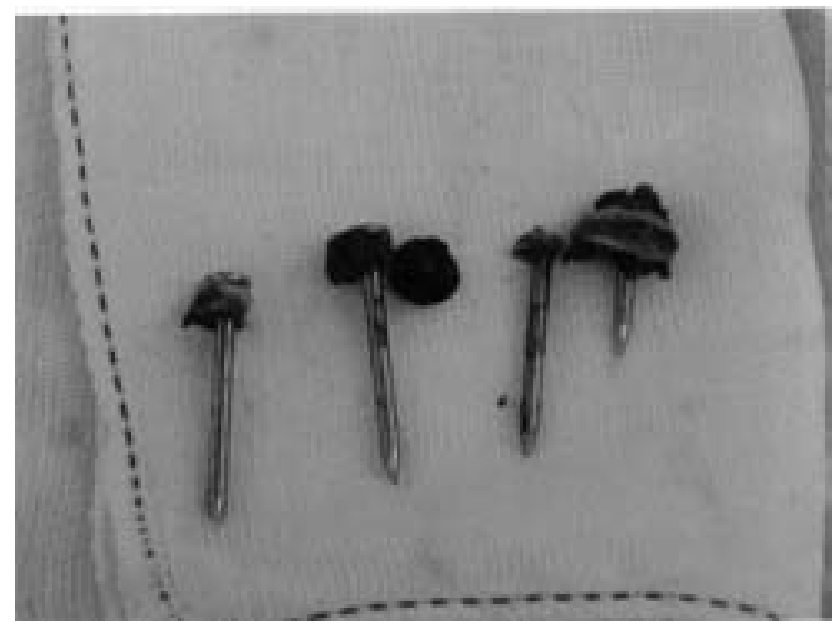

Figura 6. Los clavos una vez extraídos: tres de $4 \mathrm{~cm}$ y uno de $2 \mathrm{~cm}$.

que fue impactado (Fig. 6). Tras retirada de cada uno de los clavos se comprobó ausencia de sangrado a través del orificio dural y se colocó hemostático sobre el mismo, quedando un defecto óseo similar al tamaño de un trépano en el orificio de entrada de cada uno de los clavos. Se procedió a cierre habitual y a sutura cutánea de los orificios de entrada. El paciente despertó con un GCS de 15 puntos y sin déficits neurológicos. No hubo complicaciones infecciosas ni crisis comiciales postoperatorias. Se practicó Rx de cráneo y TAC de control que mostraron un defecto óseo a nivel de trayectoria de cada clavo sin restos de material metálico y ausencia de lesiones cerebrales. A los 5 meses del episodio el paciente se encuentra neurológicamente asintomático y en seguimiento por el servicio de psiquiatría tras ser diagnosticado de trastorno de personalidad.

\section{Discusión}

\section{Epidemiología}

La literatura recoge muy pocos casos de PBI producidas por pistola de clavos, siendo la mayor parte de ellas a nivel de extremidades, secundarias a un único clavo y de carácter accidental ${ }^{5}$. En total se han encontrado referencias a unos 30 casos de PBI por pistola de clavos ${ }^{5,7,9,10-13,16-}$ 18,20,23,24,26,30, siendo excepcionales los intentos de suicidio a través de este mecanismo lesivo ${ }^{29}$. En algunos de estos casos autolíticos el número de clavos fue elevado ${ }^{21}$, siendo el máximo número de clavos intracraneales notificado de once, de $8 \mathrm{~cm}$ de longitud y con cabeza, atravesando algunos de ellos por completo el diploe ${ }^{22}$.

Sólo hay dos casos recogidos en la literatura de clavos introducidos simultáneamente a nivel craneal y torácico, ambos debidos a intentos de suicidio ${ }^{14}$. No hemos encontrado ningún otro caso en la literatura de PBI por pistola de clavos en el que se hayan encontrado intracranealmente clavos de distinta índole.

Las PBI suelen producirse en aquéllas regiones donde el diploe es más fino como la superficie orbitaria y la escama del temporal. Las heridas de entrada en esta localización tienden a producir mayores déficits neurológicos debido a su menor distancia del tronco cerebral y estructuras vasculares profundas.

\section{Diagnóstico}

El diagnóstico precoz de estas lesiones es muy importante de cara al pronóstico. Muchas veces el diagnóstico se ve dificultado por la imposibilidad de obtener un relato conciso de lo sucedido por la falta de colaboración del paciente, su inconsciencia o la falta de testigos. En estos casos es imprescindible la sospecha clínica de PBI de cara a encaminar las exploraciones para confirmar dicho diagnóstico. En todos los casos de TCE leve acompañado de heridas cutáneas, es imprescindible la realización de una anamnesis exhaustiva y una Rx de cráneo $^{6}$. La Rx de cráneo es diagnóstica en la mayor parte de los casos, ya que habitualmente se trata de objetos radiopacos. Sin embargo, también debe practicarse una TC cerebral para cuantificar la extensión del daño cerebral, localizar el cuerpo extraño y valorar potenciales alteraciones de las estructuras vasculares, existencia de hemorragias y/o edema tisular. Este estudio debe incluir siempre una ventana ósea, para evaluar la presencia de fracturas e impactación de fragmentos óseos en el interior del parénquima siguiendo la trayectoria del cuerpo extraño. La RMN está contraindicada en presencia de cuerpos extraños metálicos intracraneales.

Cuando se sospecha la existencia de una lesión vascular debe practicarse una angiografía y/o una angio-TC, lo que permitirá diagnosticar disecciones arteriales, pseudoaneurismas traumáticos y fístulas arteriovenosas ${ }^{27}$. Se recomienda la realización de estas pruebas en casos de heridas en región pterional y orbitofacial y en casos con hematoma o hemorragia subaracnoidea (HSA) en la $\mathrm{TC}^{1}$. En nuestro caso no se practicaron estas exploraciones por no existir signos de sospecha de patología vascular. La incidencia de aneurisma postraumático tras PBI se sitúa entre el 3\% y el 33\% dependiendo de cuándo se realice la angiografía, con una media de 16 y 2 días tras el traumatismo respectivamente ${ }^{13}$. Sin embargo, una única angiografía negativa no descarta la posibilidad de que aparezca un aneurisma postraumático más tardío ${ }^{1}$. En su evolución natural los aneurismas postraumáticos pueden desaparecer, cambiar de tamaño o romperse ${ }^{1}$. Cuando se diagnostica un aneurisma o una fístula arteriovenosa postraumática se recomienda tratarlos 9 . Existen al menos dos casos de aneurisma postraumático asociado a PBI por pistola de clavos curiosamente, ambos casos fueron intencionales ${ }^{19,21}$. 


\section{Tratamiento}

El tratamiento de estos pacientes incluye las medidas generales de reanimación, estabilización y control de la PIC. No existen datos suficientes en la literatura para estandarizar un protocolo de tratamiento quirúrgico en las PBI, por lo que el manejo actual de estos pacientes se sustenta sólo en "recomendaciones" (grado C de evidencia científica $)^{25}$. Las principales indicaciones de tratamiento quirúrgico en PBI son:

1) evacuación de hematomas epidurales, subdurales e intraparenquimatosos; 2) desbridamiento de todos los tejidos afectados en la trayectoria para evitar infecciones y del cerebro necrótico para evitar edema e isquemia; 3 ) controlar un sangrado activo y 4) retirar fragmentos óseos o cuerpos extraños intracraneales para prevenir infecciones, epilepsia o su migración a otros compartimentos.

En heridas por arma de fuego la bala debe ser extraída si se encuentra accesible y esta maniobra no entraña un riesgo añadido para el paciente. No se recomienda desbridamiento quirúrgico agresivo del trayecto de la bala en aquellos casos sin efecto de masa significativo, ni la reintervención con la única intención de evacuar fragmentos óseos o de metralla retenidos (evidencia clase III) ${ }^{25}$. En casos de objeto penetrante, los pacientes, en los cuales dicho objeto permanece en su sitio tras la inserción, tienen menor mortalidad que aquéllos en los que es retirado antes de su llegada a un centro hospitalario $(11 \% \text { frente al } 26 \%)^{28}$. La retirada de estos objetos no debe realizarse fuera de quirófano, siendo recomendable realizar una apertura de la duramadre y disponer de una correcta visión del campo. En nuestro caso, la retirada se realizó sin craniectomía ni apertura dural previa, ya que se consideró que el riesgo de sangrado era bajo, puesto que la TC cerebral no mostró hematomas ni signos de afectación de trayectos vasculares importantes. Se consideró que en caso de producirse una hemorragia durante la retirada de los clavos, ésta seria de cuantía escasa con lo que habría tiempo de practicar craniectomía de urgencia.

Se practicó un cierre dural cuidadoso para prevenir fístulas de LCR e infecciones. Se estima que la incidencia de infección asociada a PBI es del $11 \%{ }^{3}$. De cara al pronóstico final, es importante realizar un diagnóstico precoz y un adecuado tratamiento de estas infecciones. La literatura no muestra evidencia suficiente para protocolizar el uso de antibioterapia profiláctica en las PBI, si bien se recomienda (evidencia clase III) la administración de antibioterapia de amplio espectro mantenida durante varios días después de la cirugía ${ }^{3}$. En nuestro caso no hubo en ningún momento datos clínicos de infección asociada a PBI y se utilizó como antibioterapia profiláctica una cefalosporina de tercera generación durante una semana.

La incidencia de crisis epilépticas tras PBI por arma de fuego se sitúa entre el 30 y el $50 \%$ de los casos frente a una incidencia de $3 \%$ - $17 \%$ en TCE cerrado moderado y grave $^{4}$. Respecto a la profilaxis anticomicial la literatura tampoco muestra evidencia suficiente para estandarizar su uso en las PBI, si bien se recomienda (evidencia clase III) el uso de fenitoína a dosis inicial de $15-18 \mathrm{mg} / \mathrm{kg}$ iv y continuar una semana a dosis de $200 \mathrm{mg}$ iv cada $12 \mathrm{~h}$, si el paciente no desarrolla crisis ${ }^{4}$. En nuestro caso no se utilizó tratamiento profiláctico anticomicial y el paciente no ha desarrollado crisis hasta el momento actual.

\section{Pronóstico}

Entre los factores de mal pronóstico de las PBI se encuentran una puntuación baja en la GCS a la recogida del paciente, intento de suicidio, heridas transfixiantes, PIC elevada, lesiones bihemisféricas, hemorragia intraventricular, subaracnoidea o hematoma mayor de $15 \mathrm{~mL}$, efecto de masa y desplazamiento de línea media.

En el caso que presentamos ninguno de los clavos llegó a penetrar por completo en el parénquima cerebral, quedando la cabeza impactada en el diploe. Sin duda la longitud de los clavos y el grado de penetración en el parénquima intervienen en el pronóstico de este tipo de PBI, ya que a mayor longitud y mayor penetración en el parénquima existe mayor riesgo de daño vascular y neurológico. No parece fácil determinar qué factores influyen en que la cabeza de los clavos atraviese o no el diploe por completo. En nuestro caso resulta sorprendente que ninguno de los clavos llegase a penetrar por completo en el parénquima cerebral, quedando el extremo de la cabeza de todos ellos impactando en el diploe, ya que se dispararon sobre regiones de menor grosor diploico (frontal y temporal). Además, el paciente utilizó clavos de diferente longitud (tres de $4 \mathrm{~cm}$ y uno de 2 $\mathrm{cm}$ ) y de distinto tipo (se encontraron intraoperatoriamente dos con remaches de goma alrededor de la cabeza, uno sin remache y uno descabezado). A posteriori el paciente reconoció haberlos manipulado intencionadamente.

Respecto al GCS a la recogida, nuestro paciente presentó un GCS de 15 puntos, lo cual sin duda fue determinante en la actitud quirúrgica a seguir y en el pronóstico. El pronóstico es mejor a mayor GCS inicial. Los pacientes con GCS menor de 5 tienen una mortalidad superior al $90 \%{ }^{2}$. Los pacientes con GCS mayor de 9 tienen una recuperación aceptable en más del $50 \%{ }^{31}$. Está ampliamente aceptado que el tratamiento quirúrgico agresivo puede beneficiar a aquellos pacientes con GCS superior a 8, aunque por otro lado no se recomienda dicha intervención en aquellos pacientes con GCS inferior a 5. A pesar de la existencia de estudios a favor de tratamiento quirúrgico agresivo en pacientes con un GCS $>5$ a la recogida ${ }^{8}$, existen controversias en la práctica sobre la actuación a seguir en pacientes con puntuaciones en la GCS entre 6 y $8^{15}$. No será posible protocolizar el manejo de las PBI hasta que no se consiga una clasificación y estra- 
tificación multivariable de los pacientes que haga posible llevar a cabo un estudio prospectivo multicéntrico y con distribución aleatoria de los pacientes, con la dificultad que ello implica dada la baja frecuencia de estas lesiones.

\section{Bibliografía}

1. Aarabi, B.: Management of traumatic aneurysms caused by high-velocity missile head wounds. Neurosurg Clin North Am. 1995; 6: 775-797.

2. Aldrich, E., Eisenberg, H., Saydjari, C., et al.: Predictors of mortality in severely head-injured patients with civilian gunshot wounds: A report from the NIH Traumatic Coma Data Bank. Surg Neurol 1992; 38: 418-423.

3. Antibiotic prophylaxis for penetrating brain injury. $\mathrm{J}$ Trauma 2001; 51: S34-40.

4. Antiseizure prophylaxis for penetrating brain injury. J. Trauma 2001; 51: S41-S43.

5. Beaver, A.C., Cheatham, M.L.: Life-threatening nail gun injuries. Am Surg 1999; 65: 1113-1116.

6. Boodhoo, H., Nathoo, N.: Is skull radiography necessary for open scalp wounds in minor head injuries? S Afr J Surg 2001; 39: 112.

7. Edlich, R.F., Silloway, K.A., Rodeheaver, G.T., Morgan, R.F., Birk, K., Thacker, J.G.: Industrial nail gun injuries. Compr Ther 1986; 12: 42-46.

8. Grahm, T.W., Williams, F.C. Jr, Harrington, T., Spetzler, R.F.: Civilian gunshot wounds to the head: a prospective study. Neurosurgery 1990; 27: 696-700.

9. Haddad, F.S., Haddad, G.F., Taha, J.: Traumatic intracranial aneurysm caused by missiles: their presentation and management. Neurosurgery 1991; 28: 1-7.

10. Homoe, P., Nielsen, P.M.: Intracranial injury caused by a nail gun. Safety measures in handling pneumatic tools. Ugeskr Laeger 1987; 149: 916-917.

11. Inoha, S., Suzuki. S., Fukui. M., et al.: A penetrating cranio-facial injury due to a nail-gun accident. No Shinkei Geka 1997; 25: 635-639.

12. Jensen, L.J., Voldby, B.: Brain injury caused by a nail gun. Ugeskr Laeger 1979; 141: 2968-2969.

13. Jinkins, J.R., Dadsetan, M.R., Sener, R.N., Desai, S., Williams, R.G.: Value of acute phase angiography in the detection of vascular injuries caused by gunshots wounds to the head: analysis of 12 cases. AJR Am J Roentgenol 1992; 159: 365-368.

14. Jithoo, R., Govender, S.T., Nathoo, N.: Penetrating nail gun injury of the head and chest with incidental pericallosal artery aneurysm. S Afr Med J 2001; 91: 316-317.

15. Kaufinan, H.H., Schwab, K., Salazar, A.M.: A national survey of neurosurgical care for penetrating head injury. Surg Neurol 1991; 36: 370-377.

16. Luotonen, J.: Intracranial penetration of a nail from nailing gun through cheek and infratemporal fossa (a case report). J Laryngol Otol 1986; 100: 247-250.

17. North, J.B.: Nail-gun injuries on the brain. Med J Aust. 1970; 2: 183-185.

18. Okada, T., Futami, K., Mukai, H., Ikeda, K., Yamashita, J.: Penetrating injury of the transverse sinus by a nail-gun-case report. Neurol Med Chir (Tokyo) 1993; 33: 703-705.

19. Rezai, A.R., Lee, M., Kite, C., Smyth, D., Jafar, J.J.: Traumatic posterior cerebral artery aneurysm secondary to an intracranial nail: case report. Surg Neurol 1994; 42: 312315.

20. Richardson, K.: Nail-gun injuries of the brain. Med J Aust 1970; 2: 754-755.

21. Sakuta, Y., Arai, S.: Penetrating brain injury and traumatic aneurysm caused by a nail gun. No Shinkei Geka 1997; 25: 357-362.

22. Salar, G., Costella, G.B., Mottaran, R., Mattana, M., Gazzola, L., Munari, M.: Multiple craniocerebral injuries from penetrating nails. J Neurosurg 2004; 100: 963.

23. Scarfo, G.B., Mariottini, A., Palma, L.: Oculocerebral perforating trauma by foreign objects: diagnosis and surgery. $\mathrm{J}$ Neurosurg Sci 1990; 34: 111-116.

24. Shibuya, T., Kushi, H., Miyagi, A., Miyagami, M., Tsubokawa, T.: A case of penetrating head injury caused by nail-gun. No Shinkei Geka 1993; 21: 373-377.

25. Surgical Management of Penetrating Brain Injury. J Trauma 2001; 51: S16-S25.

26. Thomas, M.D., Siu, K.: An unusual cranial injury caused by an industrial nail-gun. Med J Aust 1987; 147: 602-603.

27. Vascular complications of penetrating brain injury. J Trauma 2001; 51: S26-S28.

28. Vinas, F.C., Pilitsis, J.: Penetrating head trauma. Disponible en: http://www.emedicine.com/med/topic2888.htm (consultado el 19/05/2005).

29. Viswanathan, R., MacArthur, D.C., Whittle, I.R.: Nail gun injury to the brain: an unusual case of suicide. Scott Med J 1994; 39: 83.

30. Wu, W.Q., Tham, C.F., Oon, C.L.: Cranio-cerebral injuries from nail-gun used in the construction industry. Surg Neurol 1975; 3: 83-88.

31. Zafonte, R.D., Wood, D.L., Harrison-Felix, C.L., Valena N.V., Black, K.: Penetrating head injury: a prospective study of outcomes. Neurol Res 2001; 23: 219-226.

Zazpe. I.; Vázquez, A.; Beaumont, C.; Bardón, A.; Azcona, J.; Gallo-Ruiz, A.; Portillo, E.: Heridas múltiples penetrantes intracraneales causadas por pistola de clavos: caso clínico. Neurocirugía 2006; 17: 544-549.

Correspondencia postal: Idoya Zazpe. Servicio de Neurocirugía. Hospital de Navarra. Irunlarrea 3. 31008 Pamplona (Navarra). 